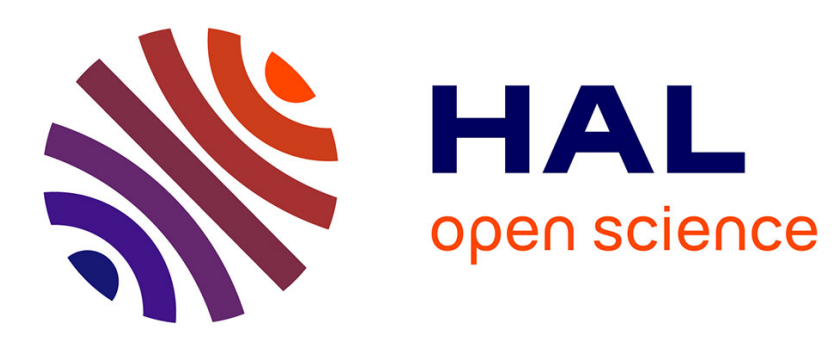

\title{
Contrasting stochasticity with chaos in a permutation Lempel-Ziv complexity - Shannon entropy plane
} Diego Mateos, Steeve Zozor, Felipe Olivares

\section{To cite this version:}

Diego Mateos, Steeve Zozor, Felipe Olivares. Contrasting stochasticity with chaos in a permutation Lempel-Ziv complexity - Shannon entropy plane. Physica A: Statistical Mechanics and its Applications, 2020, 554, pp.124640. 10.1016/j.physa.2020.124640 . hal-03008566

\section{HAL Id: hal-03008566 https://hal.science/hal-03008566}

Submitted on 16 Nov 2020

HAL is a multi-disciplinary open access archive for the deposit and dissemination of scientific research documents, whether they are published or not. The documents may come from teaching and research institutions in France or abroad, or from public or private research centers.
L'archive ouverte pluridisciplinaire HAL, est destinée au dépôt et à la diffusion de documents scientifiques de niveau recherche, publiés ou non, émanant des établissements d'enseignement et de recherche français ou étrangers, des laboratoires publics ou privés. 


\title{
Contrasting stochasticity with chaos in a permutation Lempel-Ziv complexity - Shannon entropy plane
}

\author{
Diego M. Mateos ${ }^{\mathrm{a}, \mathrm{b}, *}$, Steeve Zozor ${ }^{\mathrm{c}}$, Felipe Olivares $^{\mathrm{d}}$ \\ ${ }^{a}$ Facultad de Ciencia y Tecnología, Universidad Autónoma de Entre Ríos. Paraná, Argentina. \\ ${ }^{b}$ Instituto de Matemática Aplicada del Litoral (IMAL-CONICET-UNL). Santa Fe, Argentina. \\ ${ }^{c}$ CNRS, GIPSA-Lab, Univ. Grenoble Alpes, France. \\ ${ }^{d}$ Instituto de Física, Pontificia Universidad Católica de Valparaíso (PUCV), 23-40025 Valparaíso, Chile.
}

\begin{abstract}
This paper aims at introducing the Lempel-Ziv permutation complexity vs. permutation entropy plane (EC-plane) as a tool to analyze time series with diverse dynamical nature. These two quantities use the Bandt and Pompe representation to quantify a continuous-state time series. The main strength of this approach lies in the fact that this plane combines two different perspectives to study a signal, one being purely statistic (the permutation entropy) and the other being algorithmic (the Lempel-Ziv complexity). The results allow us to conclude that the EC-plane constitutes an appropriate framework for: (i) characterizing non-linear chaotic maps, (ii) distinguishing deterministic from stochastic processes and (iii) to discriminate between fractional Brownian motion, fractional Gaussian noise and $K$-noise.
\end{abstract}

Keywords: Chaos, Noises, Entropy, Complexity, FBM, FGN.

\section{Introduction}

Signals obtained from real physical experiments are very often produced by coupled systems and exhibit complex dynamics. In addition to their deterministic behavior due to the underlying physical structure, there is very often a stochastic component coming from diverse noise sources $[1,2]$. H. Wold showed in $[1,2]$ that any stationary time series can be decomposed into two different parts: i) a deterministic one where the state of the series at a given time is a function by its own past, and ii) a finite order moving average component. In various applications, the question of deciding whether the deterministic part of the signal is dominant, of regular or chaotic nature, or if it is mainly stochastic is crucial for its comprehension and analysis.

To address these issues, signals are usually analyzed using tools coming either from the probability theory or from the nonlinear dynamics. The former approach includes the

\footnotetext{
*Corresponding author

Email addresses: mateosdiego@gmail.com (Diego M. Mateos), steeve.zozor@gipsa-lab.grenoble-inp.fr (Steeve Zozor), olivaresfe@gmail.com (Felipe Olivares) 
analysis of statistics of the data ( e.g., probability distribution, higher order moments,...), analysis of the spectral content of the signals, among others. In this line, tools coming from the information theory take a particular importance $[3,4,5,6]$. On the other hand, the latter approach is well suited for signals having a deterministic origin, generally nonlinear. Tools used here are usually those describing chaotic signals, such as fractal dimensions or Lyapunov exponents, among many others (see e.g. [7, 8]). Of particular interest are the tools issued from the Kolmogorov complexity or algorithmic complexity theory [3, 9, 10, 11].

Motivated by the fact that a single measure is generally insufficient to resume the complexity (in a statistical and/or deterministic sense), it has been proposed in the literature to analyze time series using simultaneously two measures of information in a so-called "information plane, demonstrating that the joint use of two quantities gives a richer information about the series than each measure separately [12]. Such an approach has been used in the literature in various areas, for example, to differentiate chaotic series from random signals [13], to characterize chaotic maps [14], to determine the authorship of literary texts [15], to quantify the crypto currencies prices inefficiency [16], or for the analysis of the EEG dynamics [17]. In these mentioned works, the informational measures used were based on statistical measures, for instance, the "Martin-Plastino-Rosso (MPR) statistical complexity $[18,19]$. However, the statistical measures are not always able to capture important characteristics of a time series. As an example, the Shannon entropy or statistical complexity usually make difficult to distinguish persistent from anti-persistent behavior in fractional Gaussian noises [13]. To overcome such a drawback, in addition to a statistical measure, we propose to employ a measure based on the algorithmic complexity with the idea to extract complementary information to a statistical one. More precisely, in this work, we propose to use simultaneously a statistical and an algorithmic measure, namely the well known Shannon entropy and the Lempel-Ziv complexity [20, 21], to analyse times series and in particular to contrast stochasticity with chaos. By this way, when analyzing time series, it is expected to capture both the random informational content and the deterministic one. This approach differ substantially from the various variants of the literature $[12,13,15,22,14,17]$.

By definition of these quantities, the use of the Lempel-Ziv complexity as well as of the Shannon entropy, one has to deal with time series taking their values over a discrete finite size alphabet ${ }^{1}$. Thus, the quantization of a signal, i.e., to convert a continuous-states signal into a discrete-states one, is a fundamental issue of the analysis. Many methods to quantize continuous-states data exist in the literature, from nonparametric estimators making use of the nearest neighbors or graph lengths for instance [23, 25, 24, 26, 27], to quantifiers based on Parzen-Rosenblatt approaches, for instance, with a square kernel, i.e., using histograms [28, 29]. In this paper, we focus on an approach proposed by Bandt and Pompe introduced a decade ago, based on a multivariate trajectory build from the scalar series, i.e., an embedding, and the so-called permutation vectors constructed from this embedding [30].

\footnotetext{
${ }^{1}$ Actually, the "entropy of continuous-state random variables is defined by analogy with the entropy and is called differential entropy. However, in practice, the estimation of this differential entropy from the data is not an easy task $[23,24]$.
} 
More precisely, for each point of the embedded trajectory, the vectors components are sorted in ascending order, and the values are replaced by their rank. The probability distribution of the permutation vectors is thus used to evaluate the Shannon entropy, i.e., the so-called permutation entropy [30]. In the same context, we proposed recently the use of the LempelZiv complexity applied to permutation vectors for the continuous-states sequences, leading to what we named permutation Lempel-Ziv complexity [31]. In the present paper, we propose to contrast deterministic with stochastic dynamics by using the hence constructed entropycomplexity plane.

The paper is organized as follows. Section 2 gives an introduction to the measures used for the analysis. Section 3 describes the permutation method we used to quantize the series, i.e., the procedure to obtain the permutation vectors. In Section 4 we analyzed with the entropy-complexity plane chaotic maps, Gaussian and non-Gaussian noises. Finally, some discussions are given in Section 6.

\section{Definitions of the uncertainty measures considered in the study}

A great number of measures were proposed in the literature to analyse time series; for example, Lyapunov exponent [32], entropy [21] and complexities [13, 20]. But many times the information obtained by these quantifiers is insufficient to understand the dynamics of the series completely. To address this problem, recent research where are combined two informational measures via an entropy-complexity plane for such a discrimination has proved to be very promising [13]. Motivated by such studies, we propose here to combine the two particular quantities introduced above, namely the permutation Shannon entropy and permutation Lempel-Ziv complexity.

Esta parte es una repetición de lo que decimos en la introduccion. Hay que re-pensarlo. Entendiendo lo que dicen pero no se por que les parece mal, muchas veces las ideas se repiten para enfocar al lector, si tienen una idea de como rescribirlo, adelante

\subsection{Shannon entropy}

The concept of entropy was introduced in thermodynamics, statistical and quantum physics by Boltzman, Gibbs or von Neumann among others [33, 34, 35, 36, 37, 38, 39]. It found its counterpart in the communication domain through the seminal work of Claude Shannon of 1948 [21]. The aim of Shannon was to define a measure of uncertainty attached to discrete-states random variable under some axiomatic, namely: (i) the invariance by permutation of the probabilities attached to the random variable; (ii) an increase with the dimension of the state space when the distribution is uniform; and (iii) a recursive property (ruling the loss of entropy when joining two states into one). These so-called ShannonKhinchin axioms [40] led to the following definition of the entropy $H[X]$ of a discrete-state random variable, taking its outcomes in a discrete alphabet $\mathcal{A}$ of finite size $\alpha=|\mathcal{A}|$, with the probability mass function $p_{X}(x)=\operatorname{Pr}[X=x], x \in \mathcal{A}[21,3]$

$$
H[X]=-\sum_{x \in \mathcal{A}} p_{X}(x) \log \left(p_{X}(x)\right) .
$$


In the Shannon definition, the base 2 logarithm is used and $H$ is expressed in bits; the natural logarithm can also be used and thus $H$ is expressed in nats.

The so-called Shannon entropy is a functional of the distribution of $X$ and does not depend on the values taken by random variable $X$. It is straightforward to show that $0 \leq H[X] \leq \log |\mathcal{A}|$, where $H[X]=0$ is minimal when $p(x)=\delta_{x, x_{0}}$ for a given $x_{0} \in \mathcal{A}$ (all the information about $X$ is known) and $H[X]=\log |\mathcal{A}|$ is maximal when the distribution is uniform over $\mathcal{A}$. Thus, the $\operatorname{logarithm} \log _{\alpha}$ of base $\alpha$ should be preferred,

$$
h[X]=-\sum_{x \in \mathcal{A}} p(x) \log _{\alpha}(p(x))
$$

so that the entropy is normalized. When the dimension $d$ of $X$ is higher than one, one sometimes deals with the entropy per number of components $\frac{H[X]}{d}$ and for an infinite sequence of variable (or vector) with the so-called entropy rate which is the limit of the entropy per variable when the length goes to the infinity.

\subsection{Lempel-Ziv complexity}

The entropy and the tools associated with allow a statistical characterization of a random variable and/or of a sequence. Conversely, to such an approach, Kolmogorov introduced the notion of complexity of an observed sequence, viewed as a deterministic one (a trajectory), to be the size of the minimal (deterministic) program (or algorithm) allowing to generate the observed sequence [3, Chap. 14]. This notion is closely linked to the idea of sequence compressibility. Later on, Lempel and Ziv proposed to define a complexity restricting to the programs based on recursive copy-paste operators [20]. Their approach and variations gave rise to the well-known algorithms of compression such as the famous gzip [3, 41, 42, 43].

To be more precise, let us consider a finite-size sequence $S_{0: T-1}=S_{0} \ldots S_{T-1}$ with size $T$, where the symbols $S_{i}$ take their values on an alphabet $\mathcal{A}$ of finite size $\alpha=|\mathcal{A}|$. The definition of the first version Lempel-Ziv complexity lies in the two concepts of reproduction and production [20]:

- Reproduction: it consists in extending (or reproducing) a sequence $S_{0: T-1}$ with a sequence $Q_{0: N-1}$ only via recursive copy-paste operations, which leads to $S_{0::_{T}+N-1}=$ $S_{0: T-1} Q_{0: N-1}$, i.e., where the first letter $Q_{0}$ is in $S_{0: T-1}$, let us say $Q_{0}=S_{i}$, the second one is the following one in the extended sequence of size $T+1$, i.e., $Q_{1}=S_{i+1}$, etc.: $Q_{0: N-1}$ is a subsequence of $S_{0: T+N-2}$. All the information of the extended sequence $S_{0: T+N-1}$ is in $S_{0: T-1}$.

- Production: In this case the subsequence $S_{0: T+N-2}$ taken from the extended sequence $S_{0: T+N-1}$ can be reproduced by $S_{0: T-1}$ but the last symbol of the extension can either follow the recursive copy-paste operation (thus we face a reproduction) or can be new. Note thus that a reproduction is a production, but the converse is false. Let us denote a production by $S_{0: T-1} \Rightarrow S_{0: N+T-1}$. 
Any sequence can be viewed as constructed through a succession of productions, called a history $\mathcal{H}$. For instance, a history of $S_{0: T-1}$ can be $\mathcal{H}\left(S_{0: T-1}\right): \emptyset \Rightarrow S_{0} \Rightarrow S_{0: 1} \Rightarrow \cdots \Rightarrow$ $S_{0: T-1}$. The number of productions used for the generation, denoted here $C_{\mathcal{H}\left(S_{0: T-1}\right)}$, is here equal to the length of the sequence. For a given sequence, obviously, there is no unique history. In the spirit of the Kolmogorov complexity, Lempel and Ziv were interested in the "optimal" history, i.e., the minimal number of productions necessary to generate the sequence. The size of the shortest history is the so-called Lempel-Ziv complexity, denoted as $C\left[S_{0: T-1}\right]=\min _{\mathcal{H}\left(S_{0: T-1}\right)} C_{\mathcal{H}\left(S_{0: T-1}\right)}[20]$. In a sense, $C\left[S_{0: T-1}\right]$ describes the minimal "algorithmic information" needed to generate the sequence $S_{0: T-1}$ by recursive copy-paste operations. One use sometimes a normalized Lempel-Ziv complexity

$$
c\left[S_{0: T-1}\right]=\frac{C\left[S_{0: T-1}\right] \log _{\alpha} T}{T}
$$

as one can show that, asymptotically with the length of the sequence, $c$ goes to the interval $[0,1][20]$.

The Lempel-Ziv complexity has various properties. Among them, it is remarkably connected to the Shannon entropy rate when dealing with sequences randomly drawn: for an ergodic sequence $^{2}$,

$$
\lim _{T \rightarrow \infty} c\left[S_{0: T-1}\right]=\lim _{T \rightarrow \infty} \frac{h\left[S_{0: T-1}\right]}{T}
$$

with probability $1[20,44]$. Note that in the stationary case, i.e., when the statistics are invariant by time translation, the joint entropy being lower or equal to the sum of the individual entropies [3], the limit of the normalized Lempel-Ziv complexity is upper-bounded by the entropy of the symbols of the sequence.

\section{From continuous-state signals to discrete state representations}

As previously introduced, the aim of the present paper is to analyze time series in a complexity-entropy plane, namely the Lempel-Ziv complexity and Shannon entropy plane. However, as described above, the Lempel-Ziv complexity can be defined only for discretestates sequences. Concerning the Shannon entropy, we have also seen that even if definitions exist for both discrete-state and continuous-state, random variables; it is more adapted to the uncertainty description of discrete-state variables (definition based on an axiomatic, estimations problems in the continuous-state context).

When dealing with continuous-state data, which is the most natural case in various contexts, before any analysis, an observed sequence has to be quantized. Different methods can be envisaged, all of them having impacts on the interpretation of the uncertainty measure (entropy, complexity) associated to the hence quantized sequence (see e.g., [45, 31]). We focus here on an approach based on the so-called permutation vector, which was at the heart of the so-called permutation entropy proposed by Bandt and Pompe [30].

\footnotetext{
${ }^{2} \mathrm{~A}$ sequence $S_{0: T-1}$ is said ergodic if, for any function $g, \sum_{k \geq 0} g\left(S_{k}\right)$ converges (almost surely) to a deterministic value.
} 


\subsection{Permutation vectors}

The Bandt and Pompe approach seems to take its origin in the study of chaos [30], and more specifically through the famous Taken's delay embedding theorem [46]. The core of this theorem concerns the reconstruction of the state trajectory of a dynamical system from the observation of one of its states. To fix the ideas, consider a real-valued discrete-time series $\left\{X_{t}\right\}_{t \in \mathbb{N}}$ and two integers $d \geq 2$ and $\tau \geq 1$, and from the series, let us then define a trajectory in the $d$-dimensional space, $\left\{Y_{t}^{d, \tau}\right\}_{t \geq(d-1) \tau}$, as:

$$
Y_{t}^{d, \tau}=\left[\begin{array}{llll}
X_{t-(d-1) \tau} & \cdots & X_{t-\tau} & X_{t}
\end{array}\right]
$$

where dimension $d$ is called embedding dimension, and $\tau$ time delay. In the domain of chaos analysis, the Taken's theorem gives conditions on $d$ and $\tau$ such that the embedding $Y_{t}^{d, \tau}$ preserves the dynamical properties of the full dynamic of the underlying system. This point goes beyond the scope of the present paper. Therefore, we do not provide detailed information and suggest to the readers refer to Ref. [46, 47].

Now, the Bandt and Pompe idea is to map continuous-state time series into discretestates one, replacing each component of vector $Y_{t}^{d, \tau}$ by its rank when the components are sorted ( e.g., in ascending order). The discrete-state vector is called permutation vector (or pattern) and is denoted $\Pi\left(Y_{t}^{d, \tau}\right)$ in the following. Such a vector takes its values over the alphabet $\mathcal{P}$ issued from the ensemble of the permutation of $\{0, \ldots, d-1\}$, of cardinal $|\mathcal{P}|=d$ !. As an illustration, if we have the vector $Y_{1}^{3,1}=\left[\begin{array}{lll}.55 & 1.7 & -.45\end{array}\right]$, the permutation vector associated with is $\Pi\left(Y_{t}^{d, \tau}\right)=\left[\begin{array}{lll}1 & 2 & 0\end{array}\right]$. As mentioned in a series of papers $[48,49$, $50,51,14]$, the frequencies or the organization of patterns in a sequence can reveal a chaotic behavior versus a random one.

\subsection{Permutation uncertainty and complexity measures}

In their paper [30], Bandt and Pompe defined the permutation entropy as the Shannon entropy of the empirical distribution associated to the permutation vector; i.e., where the probabilities are the frequencies of each possible permutation vector in the sequence $\left\{\Pi\left(Y_{t}^{d, \tau}\right)\right\}_{t}$. When dealing with time-series issued from a random process, this is nothing more than an estimation of the Shannon entropy of the permutation vectors process. In the following, we use the terminology permutation Shannon entropy to be more precise. Moreover, to distinguish the permutation entropy as defined by Bandt and Pompe from the formal entropy of a random permutation vector, we will use the notation,

$$
\widehat{h}^{d, \tau}\left[X_{0: T-1}\right]=-\sum_{\pi \in \mathcal{P}} \widehat{f}_{X}^{d, \tau}(\pi) \log _{d !} \widehat{f}_{X}^{d, \tau}(\pi)
$$

where $\widehat{f}_{X}^{d, \tau}(\pi)$ is the proportion (frequency) of the permutation vector $\pi$ in the sequence $\left\{\Pi\left(Y_{t}^{d, \tau}\right)\right\}_{t}$. 
In a similar way to the Bandt and Pompe aproach, in a previous work [31], we proposed the Lempel-Ziv complexity of the permutation vector sequence, as a tool for the analysis of a complex time series. We named this tool permutation Lempel-Ziv complexity, which is denoted

$$
\widehat{c}^{d, \tau}\left[X_{0: T-1}\right]=c\left[\Pi\left(Y_{(d-1) \tau}^{d, \tau}\right) \cdots \Pi\left(Y_{T-1}^{d, \tau}\right)\right]
$$

in the sequel.

In the present paper, we propose to analysis time series in the hence defined permutation Lempel-Ziv complexity - permutation Shannon entropy plane, in short EC-plane, by representing a sequence $X_{0: T-1}$ by a point $\left(\widehat{h}^{d, \tau}\left[X_{0: T-1}\right], \widehat{c}^{d, \tau}\left[X_{0: T-1}\right]\right)$ in a 2D-plane. The motivations lie on the following observations:

- As noted by several authors [48, 49], in various chaotic contexts, the presence of forbidden patterns can reveal the chaotic behavior of a sequence since, in general, there is no forbidden pattern in random sequences. As an example, for the logistic

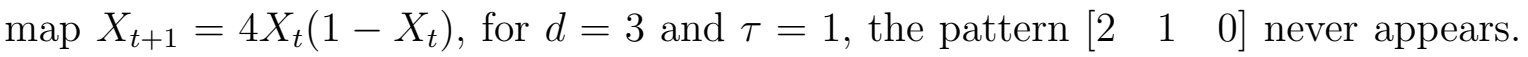
However, this is not always the case: there are chaotic maps without forbidden patterns and conversely noises exhibiting forbidden patterns [50,51]. Therefore, the chaotic aspect can be revealed by the time organization of the permutation vectors by their frequencies of occurrence; i.e., by the permutation Lempel-Ziv complexity rather than by the permutation Shannon entropy.

- From the relations Eqs. (2) for (sufficiently long) stationary and ergodic random sequences, the normalized complexity reaches the entropy rate of the sequence. From the fact that for random variables $X_{i}, H\left(X_{1}, \ldots, X_{n}\right) \leq \sum_{i} H\left(X_{i}\right)$ [3], the entropy rate is always less than individual entropies. In the other word, one may expect that $\widehat{c}^{d, \tau} \leq \widehat{h}^{d, \tau}$, with equality for sequences of permutation vectors with independent and identically distributed samples. This results in the existence of a straight line, where no signal can be above.

- Moreover, if in a parametrized family of noise series, the entropy rate (of the permutation vectors) is linked to the individual entropy through a function of the parameter, the noises values $\left(\widehat{h}^{d, \tau}, \widehat{c}^{d, \tau}\right)$ are expected to be close to this straight line.

- From the previous remark, one may expect that in chaotic sequences, for a given permutation entropy, the complexity will be lower than for noisy sequences due to the temporal organization governed by a deterministic dynamics.

In other words, it is expected that various kinds of noises and of chaos can be better characterized (separated) in such an EC-plane, distinguishing in some sense the part of algorithmic complexity and the part of statistical uncertainty contained in a time series. This substantially differs from the variants of information plane proposed in the literature $[12,13]$.

Finally, note that the proposed analysis of a series applies as well when dealing with intrinsic vector series. In this case, as done in [31], the permutation vectors are issued from 
the vector of the trajectories (and thus no embedding is done previously to permutation procedure).

Let us now turn to the various type of time series we aim at studying with the proposed approach.

\section{Characterization of chaotic maps and noises}

To illustrate how the permutation Lempel-Ziv complexity vs permutation Shannon entropy plane can reveal characteristics of a time series through this EC-plane, we analyze chaotic and random series which will describe in the next subsections. Our purpose is to exhibit that the proposed plane allows not only to distinguish between stochastic and chaotic time series but also to separate Gaussian and non-Gaussian processes with the same spectrum over their "degree of correlation. In the sequel, the number in parentheses will denote the studied map and only this number is reported in the figures.

\subsection{Chaotic maps}

In the present work, we consider 26 chaotic maps described by Sprott in the appendix of his book [52]. These chaotic maps are grouped as follows.

- Conservative Maps: conservative systems have some conserved quantities, such as mechanical energy or angular momentum. In this case, the phasespace volume is preserved. In this paper, we analyze the following conservative maps (1) Arnold's cat map, (2) the chaotic web map, (3) the Chirikov standard, (4) the Gingerbreadman, (5) the Hénon area-preserving quadratic, (6) the Lorenz three-dimensional chaotic map,

- Dissipative maps: mechanical systems are systems in which mechanical energy is converted (or dissipated) into heat. A consequence is that the phasespace volume contracts [52]. In this paper, we analyzed the following dissipative maps: (7) The Hénon map, (8) the Lonzi map, (9) the Delayed logistic map, (10) the Tinkerbell map, (11) the Holmes cubic map, (12) the dissipative standard map, (13) the Ikeda map, (14) the Sinai map, (15) the discrete predator prey map.

- Non-inverted maps: An iterated map is called noninverted, when in a sequence, each iterate $X_{n}$ has two preimages $X_{n-1}$ that do not coincide. Consequently, one it of information (a factor of 2) is lost with each iteration since there is no way to know from which preimage each value came. This exponential loss of information is equivalent to exponential growth of the error in the initial condition that is the hallmark of chaos [52]. Here, we analyzed the following non inverted maps, (16) The lineal congruential generator, (17) the cubic map, (18) the Cusp map, (19) the Gauss map, (20) the logistic map, (21) Pincher's map, (22) Ricker's population model, (23) the sine circle map, (24) the sine map, (25) the Spence map, (26) the tent map. 
For all the maps presented above, we use the parameters and initial conditions expressed in [52]. For more detail about each map see [52, 13, 14].

\subsection{Random sequences}

For the study of random sequences we used the following Gaussian and nonGaussian, stationary or nonstationary noises:

- K-noises with a power spectrum $1 / f^{k}$. Noises with such a power-law spectrum are widely found in nature, in physics [53], in biology [54], in astrophysics [55] among other domains. Such noises are not necessarily Gaussian. In particular, in this paper, we focus on noises generated through the algorithm described in [13] that basically consists in (i) generating a pseudo random sequences of independent samples with uniform probability distribution and zero mean value, (ii) taking the Fourier transform, (iii) multiplying this Fourier transform by $f^{-k / 2}$ and symmetrizing the result to obtain a real function (iv) performing the inverse Fourier transform and discarding the small imaginary components produced by numerical approximations. The obtained sequence are nonGaussian [13]. In this work we concentrate on the parameters $k=0.25 \times n, n=$ $0, \ldots 14$, cases respectively denoted (27) to (41).

- Standard fractional Brownian motion (FBM). The Gaussian process $B_{H}(t)$ is nonstationary and parametrized by a quantity $H \in(0,1)$, called Hurst exponent, and has the covariance function $\operatorname{Cov}_{B_{H}}(t, s)=\mathrm{E}\left[B_{H}(t) B_{H}(s)\right]$

$$
\operatorname{Cov}_{B_{H}}(t, s)=\frac{1}{2}\left(|t|^{2 H}+|s|^{2 H}-|t-s|^{2 H}\right) .
$$

This process was introduced by Kolmogorov [56] and studied by the climatologist Hurst [57] and later on by Mandelbrot and Van Ness in [58] to model fractals for instance. The FBM increments $B_{H}(t)-B_{H}(s)$ are stationary and the process is selfsimilar, i.e., $B_{H}(a t)$ has the same distribution as $|a|^{H} B_{H}(t)[59,60,61,62,63$, and ref. therein]. These processes exhibit a very rich behavior depending on $H$ : for $H=\frac{1}{2}$ one recovers the standard Brownian motion (limit process of the random walk); For $H>\frac{1}{2}$, the process exhibits persistency in the sense that a trend or increment sign in the past tends to persist in the future (the increments have a positive correlation) and the process exhibits long range dependence; for $H<\frac{1}{2}$, the process is anti-persistent in the sense that the trends from past to future are more likely to be opposite (the increments have a negative correlation). Finally, note that the spectrum ${ }^{3}$ of an FBM is proportional to $\frac{1}{f^{2 H+1}}[60,64]$.

- Fractional Gaussian noise (FGN). Such a process is defined as the increments of a FBM [65], as

$$
G_{H}(t)=B_{H}(t+1)-B_{H}(t)
$$

\footnotetext{
${ }^{3}$ Because the process is non stationary, rigorously the notion of spectrum has no sense in itself. However, one can consider it through the Wigner-Ville spectrum, averaged in time [60], which would be the spectrum estimated from a sample path for instance.
} 
Due to the stationarity of increments of FBM, a FGN is stationary and it is straightforward to show that its covariance function is

$$
\operatorname{Cov}_{G_{H}}(u)=\frac{1}{2}\left(|u+1|^{2 H}-2|u|^{2 H}+|u-1|^{2 H}\right) .
$$

Note that for $H=\frac{1}{2}$ the correlation function vanished for non-zero lags $u$. Thus $G_{\frac{1}{2}}$ corresponds to a Gaussian white noise. Finally, note that the spectrum of FGN is proportional to $\frac{1}{f^{2 H-1}}$ [64]. When $H \in(0.5,1), C_{G_{H}(u)}$ is positive and finite for all $u$ and the corresponding series exhibits long-range dependence. If $H \in(0,0.5)$, the integral of $\operatorname{Cov}_{G_{H}(u)}$ is zero and $C_{G_{H}(0)}$ diverge when $u \rightarrow \infty$. Hence, it is short-range dependence when $H \in(0,0.5)$. Finally, when $H=0.5$ we obtain white noise [64]. Hay un problema acá. La covarianza, asi escrita, claramente no da 0 para $u \neq 0$. Hay que mirar bien por ejemplo Moltz, donde se define $G_{H}(t, h)=\frac{1}{h}\left(B_{H}(t+h)-B_{H}(t)\right)$ da una covarianza bajo la forma $\operatorname{Cov}_{G_{H}}(\tau)=\mathrm{E}\left[G_{h}(t, h) G_{H}(t+\tau, h)\right]=\frac{1}{2 h^{2}}\left(|\tau+h|^{2 H}+|\tau-h|^{2 H}-2|\tau|^{2 H}\right)$. Ahora, para $\tau=0$ se obtiene $\operatorname{Cov}_{G_{H}}(0)=h^{2 H-2}$ que es constante cuando $H=\frac{1}{2}$. Para $\tau \neq 0$, con un desarollo de Taylor al orden 2 se muestra sencillamente que cuando $h \rightarrow 0, \operatorname{Cov}_{G_{H}}(\tau)$ tiende a $2 H(2 H-1)|\tau|^{2 H-2}$ que vale 0 para $H=\frac{1}{2}$. Hay que retomar este caso rigurosamente. Ademas, el fin del parafo no se entiende nada sobre el tema se short y longue range depndence... Steeve estuvimos discutiendo con Felipe y no entendemos, estas definiciones fueron sacadas de las bibliografias citadas. Tus comentarios superan nuestro conocimiento del tema. Si sabes como corregirlo hacelo, sino lo dejamos como esta.

To generate time series from FBM and FGN, we used the algorithm proposed by Abry and Sellan [66, 67]. By nature, the sequences generated by this algorithm are discrete-time approximation of the continuous-time sequence, which is precisely needed to be able to analyze such sequences in the plane previously introduced.

\subsection{Chaos and $K$-noises analysis}

For each of the 41 times series labeled in the previous section ((1) to (26) for the chaotic maps, and (27) to (41) for the $K$-noise) we generated $N=4 \times 10^{4}$ times series of $L=10^{6}$ samples. For each time series, we computed the corresponding sequence of permutation vectors (choosing the parameters $(d, \tau)$ ), and then the permutation Shannon entropy $\hat{h}^{d, \tau}$ and the permutation Lempel-Ziv complexity $\widehat{c}^{d, \tau}$. Figure 1A depicts the points $\left(\left\langle\hat{c}^{d, \tau}\right\rangle_{N},\left\langle\hat{h}^{d, \tau}\right\rangle_{N}\right)$ where $\langle\cdot\rangle_{N}$ denotes the averaged quantities over the $N$ independent realizations with parameters $(d, \tau)=(5,1)$. We also tested the embedding dimensions $d=4$ and 6 with $\tau=1$; the distribution of the coordinates in the EC-plane is similar for all parameter, giving a robust analysis over dimension $d$. Figure $1 \mathrm{~B}$ and $\mathrm{C}$ are zooms in a zone containing the coordinates for specific chaotic maps. The dots represent the mean values and the ellipses represent the dispersion of the values over the snapshots via the sample covariance matrix $\widehat{C}_{\widehat{h}, \widehat{c}}$ computed 
from the data, i.e., an ellipse corresponds to $\left[\begin{array}{ll}\widehat{h} & \widehat{c}\end{array}\right] \widehat{C}_{\widehat{h}, \widehat{c}}\left[\begin{array}{ll}\widehat{h} & \widehat{c}\end{array}\right]^{t}=1$. The inferior and lateral histograms depict the corresponding histograms of the values taken by each measure separately using $N=4 \times 10^{4}$ snapshots. Comparing the values belonging to the chaotic dynamics of the time series in the plane, with their complex and entropy values separately, we can observe that the plane allows a much clearer distinction than just only using one measure. This shown an improvement in the discrimination between different dynamics is successfully achieved by using a statistical and algorithmic measure in conjunction, compared to the measures used separately

For the $K$-noises, a smooth transition in the planar location is observed; the complexityentropy coordinates corresponding to these are remarkably aligned on a line, while that of the chaotic sequences separates clearly from this line. Due to the entropy rate of a process decreases with the temporal correlation, the entropy and complexity values decrease when the correlation increases in the noise giving values from $(\widehat{h}, \widehat{c})=(0.2,0.05)$ for $k=3.5$ to $(\widehat{h}, \widehat{c})=(1,0.485)$ for $k=0$. The observed alignment of the $K$-noises sequence points reveals a more or less linear dependence with $k$, so that due to the asymptotic behavior of these stationary ergodic sequences, permutation entropy and the permutation complexity show a linear dependence.

Referring to the chaotic maps, as already observed, the representative average coordinates clearly separate from the "noise-line", and are always positioned below this line. The separation from the line is indeed a consequence of the deterministic dynamics underlying such processes, mechanisms that are of relatively low complexity. Thus, for the same single entropy, chaotic sequences have a lower complexity than noise. A notable exception lies in the lineal congruential map (16) (see the insert of figure 1A). This anomaly can be explained by the pathological characteristic of this map. Indeed, sequences generated by this map are often used to generate pseudo-random sequences and share a huge number of characteristic of purely random sequences [68, Chap. 5]. Moreover, from the analysis of the correlation (in a deterministic sense), it appears that it is very small, explaining why the coordinate entropy-complexity of this map is so close to that of the Gaussian white noise.

In a deeper analysis, it can be seen that the proposed map allows to distinguish the Hénon area-preserving quadratic map (5) from the delayed logistic map (9), Pincher's map (21) from the Gingerbreadman map (4) and the Spence map (25) from the Hénon map (7), whereas these maps are less distinguishable using the plane previously proposed in the literature [14]. This clearly exhibits the benefit of using the algorithmic complexity in the analysis of these chaotic maps. The same scene is observed between chaotic maps and $K-$ noises, for example in the case of dissipative standard map (12) and the correlated noise with $(k=1.25)$. Here again these differences are rising due to the implementation of a non statistical measure, such as the Lempel-Ziv complexity, which demonstrates that the plane of analyze we propose here can be a good alternative when sequences are not separable in the plane previously proposed in the literature (and conversely).

Finally, note that the chaotic maps are relatively well separated in "clusters" regarding their classification as non inverted, dissipative and conservative. This observation suggests 
that the analysis of a sequence in a permutation Shannon entropy and permutation LempelZiv complexity plane is powerful to characterize finely the class such sequences belong to.

\subsection{K-noise, FBM and FGN analysis}

Now we focus on the EC-plane analysis for the $K$-noises, FBM and FGN time series for different values of the parameter $k$ or $H$. To this aim, we generated $N=4 \times 10^{4}$ noise series, of length $L=10^{6}$ samples each. For the non-Gaussian $K$-noises we used the parameter $k \in[0 ; 3.5]$ with step $\Delta k=0.25$. For the FBM and FGN series the Hurst exponents used were $H=0.1 \times n, n=1, \ldots, 9$ and the Bandt-Pompe symbolization parameter taken for all the series are $d=5$ and $\tau=1$. Figure 2 depicts the mean values of $(\widehat{c}, \widehat{h})$ over the realizations, for each sequence.

As observed in Figure 2, the complexity-entropy points belong to the sequences of the $K$-noises spread along the straight line. As intuitively expected, signals with low correlation stay in the high entropy and complexity values, as seen zoomed in the insert of Figure 2. This effect remains for FBM sequences, but it is no more valid for the FGN. The maximum is reached when $k=0$ which corresponds to a decorrelated noise (white noise). As the noise has more correlation, the values start to move to the medium and low area. The FBM remains in the same line that as $K$-noises but lies in an intermediate-high value of entropy and complexity. It can be seen if the distance between two consecutive Hurst exponent value $\left(\widehat{c}\left(H_{i}\right), \widehat{h}\left(H_{i}\right)\right)$ and $\left(\widehat{c}\left(H_{i+1}\right), \widehat{h}\left(H_{i+1}\right)\right)$ is shorter when the processes are antipersitence $(H<1 / 2)$.

The inner box in Figure 2 shows that the FGN is concentrated in a high region of the complexity-entropy plane. In particular, given a spectrum (power-law exponent), the three types of noise are clearly separated, which is obviously impossible by means of a usual spectral analysis. This suggests that the measures proposed here can capture more information than pure statistical (Gaussian vs non-Gaussian) or stationary saco ese parrafo por que parece que hay una confucion mia muy grande, si tienen algo que aportar adelante. No queda claro: si 2 ruidos tienen espectros diferentes, se distinguen por analysis espectral, como lo decis. Es decir que hay que comparar estos tres tipos, con el mismo exponente de la decrecencia espectral; eso es dado cuando $H_{F B M}=H_{F G N-1}$ y $K=2 H_{F G N}-1$, lo que se prodria indicar. Luego, en la figura, no veo tal correspondencia... Ejemplo, el $K=3.5$ da el mismo espectro que $H_{F G N}=2.25$ que no esta en la figura, $H_{F G N}=1.25$ que tampoco hay... Ademas, no te equivocaste de label de figura? Igual el parafo siguiente?

The FGN behaviour is illustrated in the inner box Figure 2. We can note as $\min (H, 1-$ $H$ ) increases, the absolute value of the correlation increases. This behavior is conserved in terms of permutation entropy and permutation Lempel-Ziv complexity. However, the permutation entropy given by $H$ and $1-H$ is more or less identical, that is, entropy is unable to distinguish whether correlation or anticorrelation characterizes the underlying FGN process. These results are in accordance with recent works [13, 69]. Nevertheless, the permutation Lempel-Ziv complexity can clearly distinguish between them (see the insert of Figure 2). In other words, measure captures the short-range correlation, or persistency vs anti-persistency behaviour. This effect highlights the importance of using two different 


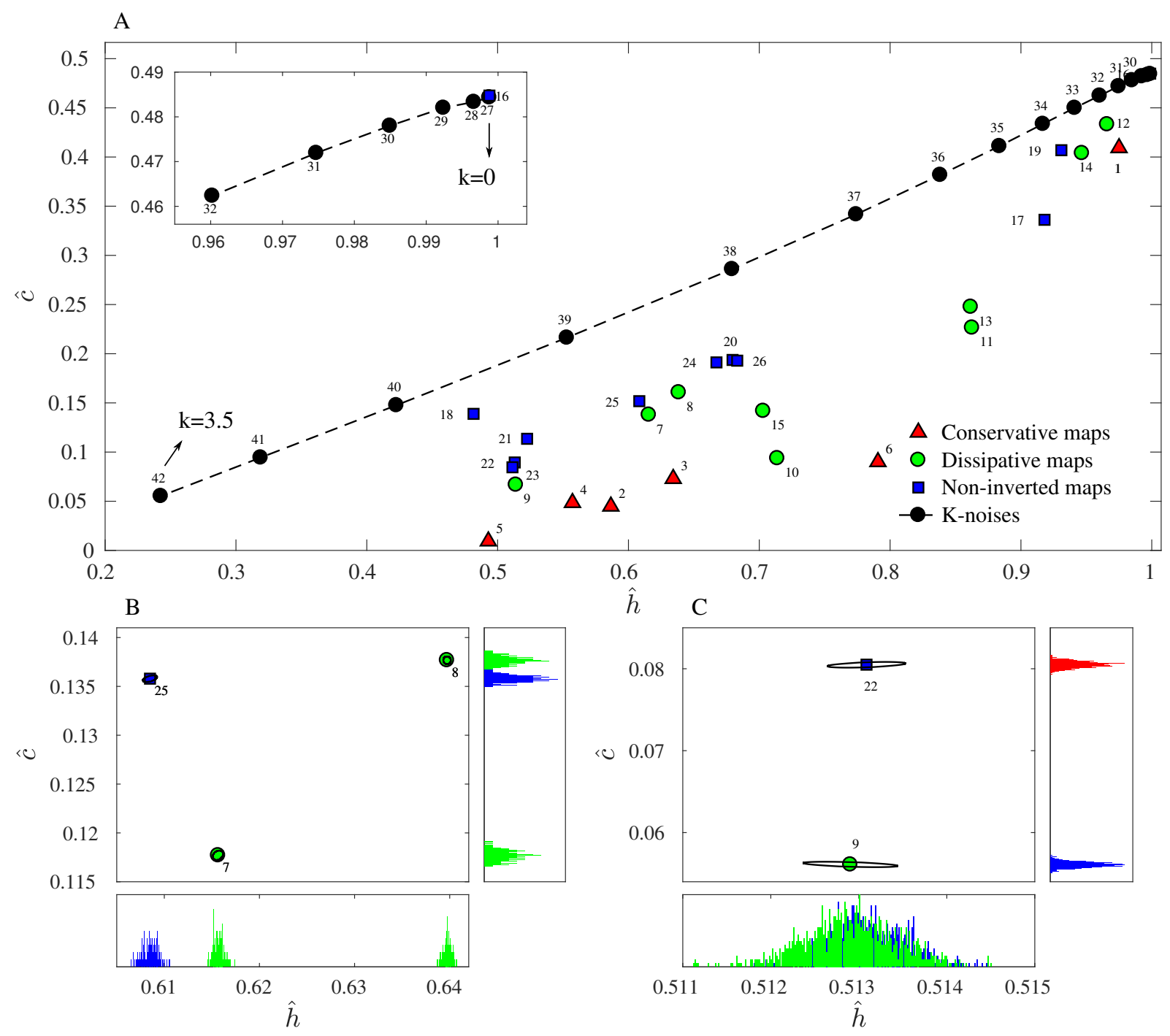

Figure 1: A) Localization in the permutation Lempel-Ziv complexity vs permutation Shannon entropy plane of the chaotic and $K$-noise series studied in the present work, for the parameters $d=5$ and $\tau=1$. For each case, we generated $N=4 \times 10^{4}$ times series of $L=10^{6}$ samples, initializing each of them randomly (see the text ) The labels correspond to those in section 4.3. All chaotic maps are clearly separate from the $K$-noises. B-C) Zoom in a zone containing the coordinates for specific chaotic maps. The inferior and lateral plots illustrate the corresponding histograms of the values taken by each measure separately using the $N=4 \times 10^{6}$ snapshots. The ellipses corresponding to the dispersion (see text). Similar results are obtained for the parameters $d=4,6$ and $\tau=1$. 
"complementary" measures to analyze such random sequences. Using this approach, we can distinguish sequences than are impossible using other maps in the literature.

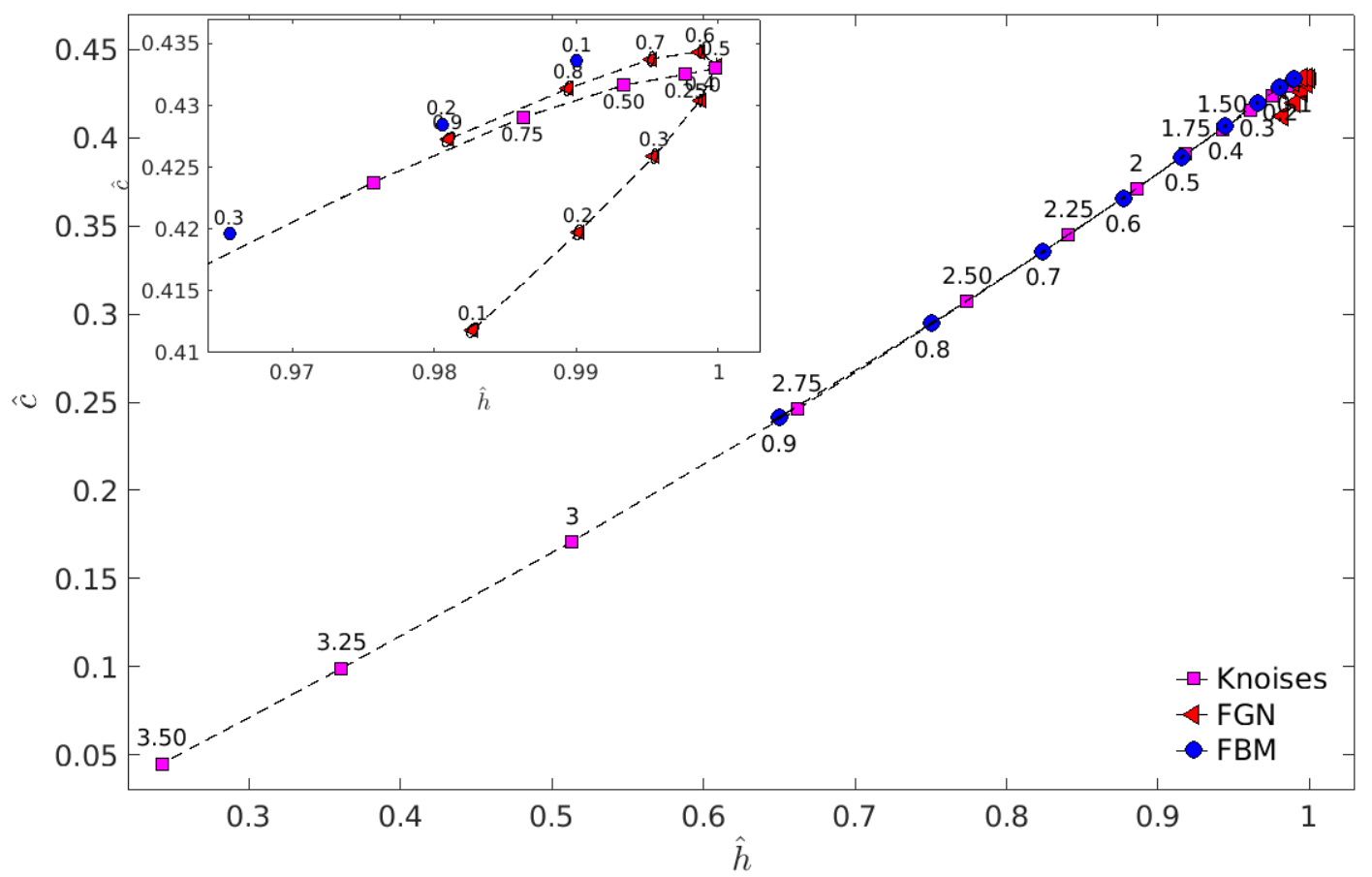

Figure 2: Localization in the permutation Lempel-Ziv complexity vs permutation Shannon entropy plane of $K$-noise, FGN and FBM sequences. The $K$-noises were generated using the parameter $M=0.25 \times m, m=$ $1, \ldots, 3.5$. For the FGN and FBM we use the Hurst exponent $H=0.1 \times n, n=1, \ldots, 9$.For each time series we generated $N=4 \times 10^{4}$ times series of $L=10^{6}$ samples. The corresponding Bandt-Pompe parameters were $d=5$ and $\tau=1$. Similar figure is obtained for parameter values $d=4,6$ and $\tau=1$.

\section{Real world data}

To illustrate the performance of the proposed representation plane in a real context, we have considered four different experimental scenarios.

- Chaotic laser data: chaotic intensity pulsations recorded from a single-mode farinfrared $\mathrm{NH}_{3}$ laser by employing a LeCroy oscilloscope. This experimental time series was used for the Santa Fe Time Series Competition. Here we have considered the longer data set (4000 data points), which presents a very clear chaotic dynamics (signal-tonoise ratio about 300) [70]. For more details of the recording procedure of this data set, please see Ref. [71].

- Stream-flow: The stream-flow process are commonly accepted as nonlinear [72]. However, it has been debated whether river flow dynamics can be chaotic or not [73, 74]. 
Here, we consider the stream-flow data of the Umqpua River, which is on the Pacific coast of Oregon in the United States. Daily stream-flow of the river starting on October 10, 1905 and ending on Jun 5, 2019, were considered (30928 data points). The data is available at United States Geological Survey website [75].

- Human gait: Hausdorff et al. have shown that the stride interval fluctuations (SIF) from subjects walking at their usual pace exhibit long-term correlations [76]. It has been shown that the SIF can be modeled as fBm with a Hurst exponent $0.88[76,77]$. The data consist of 10 sequences of SIF from different healthy subjects (available in [78]). The data lengths span in a range between 2902 and 3397 data points. Further details about the experiment and data acquisition, see Ref. [76]. Here we have analyzed the integrated SIF.

- Price returns: It is well known that prices in efficient stock markets should follow random walks [79]. Then, developed markets should produce totally independent increments of their prices. In contrast, emerging markets should exhibit temporal correlation than developed ones, suggesting more predictability and less efficiency [80, 81]. Here we have considered forty-eight stock market indices for different countries. All data were collected from the Datastream platform [82]. Daily prices starting on January 3, 2000, and ending on May 27, 2016, are considered (4,280 observations). These stock markets are classified as developed (20), emerging (17) or frontier (11) using the Morgan Stanley Capital Index. Here we have analyzed the integrated logarithmic price returns.

Entropy and complexity values for all experimental data described above are depicted in Fig. 3. Additionally, as a reference it has been included the $\mathrm{fBm}$ model locations using, in each case, the same sequence length as the experimental data. Particularly, chaotic laser data is localized underneath the stochastic reference ( $\mathrm{fBm}$ model) with medium permutation entropy and low complexity values, giving an account of its chaotic nature, as can be seen in Fig. 3A. Same affirmation stands for the stream-flow data of the Umqpua River - see Fig. 3B. From this result we can conclude that this river flow dynamics is chaotic, in accordance with results previously reported [74]. On the other hand, in Fig. 3C is observed that SIF data locates close to the fBm with $H=0.8$, as expected. Finally, results for the stock markets are depicted in Fig. 3D. It is observed that all the markets overlap with the fBm model. Developed countries tent to locate, in average, around to the random walk reference (fBm with $H=0.5$ ), while for emerging and frontier countries show long-term correlations having lower values of entropy and complexity.

\section{Discussion}

In the analysis of time series, the challenge of distinguishing chaotic from stochastic dynamics underlying a complex time series could be a critical and subtle issue. Therefore, it is important to develop methods of analysis that allow to differentiate and characterize these natures. Based on the evidence that the use of entropy-complexity planes to analyse 
CyR_RealData-eps-converted-to.pdf

Figure 3: Localization in the permutation Lempel-Ziv complexity vs permutation Shannon entropy plane of (A) chaotic laser data, (B) stream-flow data, (C) human gait dynamics and (C) price returns. fBm model locations using, in each case, the same sequence length as the experimental data are also depicted as a reference. 
time series give results allowing such discrimination $[14,13]$, in this work we introduce a particular variant of complexity-entropy plane. The main difference from the variants of the literature lie in the combination of both statistical and algorithmic complexity measures to extract both the random and deterministic parts that can compose a time series. The two chosen measures are the Shannon entropy and the Lempel-Ziv complexity applied to the permutation vectors, i.e., the so-called permutation Shannon entropy and permutation Lempel-Ziv complexity respectively. We used the hence defined EC-plane to analyze several wells-known time series of the literature -chaotic maps, $K$-noises, fractional Brownian motion and fractional Gaussian noise.

In particular, the proposed representation allows to distinguish clearly chaotic maps from random processes. Figure 4 shows that the EC-plan has the tendency to classify the chaotic maps according to their "non-inverted, "dissipative and "conservative characteristics. We can observe that there is a first area below the K-noises area (blue area) where lies all the non-inverted chaotic maps. Further down with less complexity reside most of the dissipative maps in the green area, although there are exceptions such as Sinai map (14) and dissipative standard map (12) which are in the range of the non-invertible ones. In the inferior triangular area (red) resides the conservative maps, which have lower complexity values, the only exception is the Arnolds cat map (1) which has a high entropy and complexity. This capacity to cluster chaotic maps is not obtained by other planes existing in the literature such as the entropy-complexity causal plane and causal Shannon-Fisher plane for instance [13, 14]. In addition, this plane can separate noises sharing the same spectrum, capturing implicitly both their statistics and their stationarity/nonstationarity character. The relation 2 defines a straight line in the plane as an upper-limit, i.e., where no times series generates an entropy-complexity point. The $K$-noises are distributed along this line, because for a random stationary and ergodic process, when correctly normalized, the Lempel-Ziv complexity of the sequence tends to the entropy rate of the process [3]. Because of the temporal organization governed by the deterministic dynamics in the chaotic sequences, the complexity-entropy values are far from this limit line. This gives a clear distinction between chaotic form stochastic behaviour. For those characteristics, this plane appears thus to be a good alternative or complement to the already proposed planes of the literature $[13,83]$.

Porque, de reprente, la parte que sigue aparece en la discusion? Es un analysis y deberia caer antes. Ademas, es redundante por parte con la que describe la figura 1. Sin embargo, me gusta la figura, aparte los labels que son gigantesques. Falta de organizacion. Esto es algo que me marco Rosso que hiciera para que se entendiera, mas alla que sea similar a la figura uno creo que describe bien la clusterizacin de los mapas.

Focusing on the random processes, this representation can distinguish different degrees of correlations in non-Gaussian noises. FBM and FGN processes are well separated in the permutation EC-plane. Since FGN is an increment of FBM, the entropy and complexity values are higher. Moreover, for the FGN the EC-plan can separate the long-term memory (LTM) processes from short-term memory (STM) processes, this result was not obtained using only permutation entropy [69] or other EC-plans [13]. This differentiation is given 


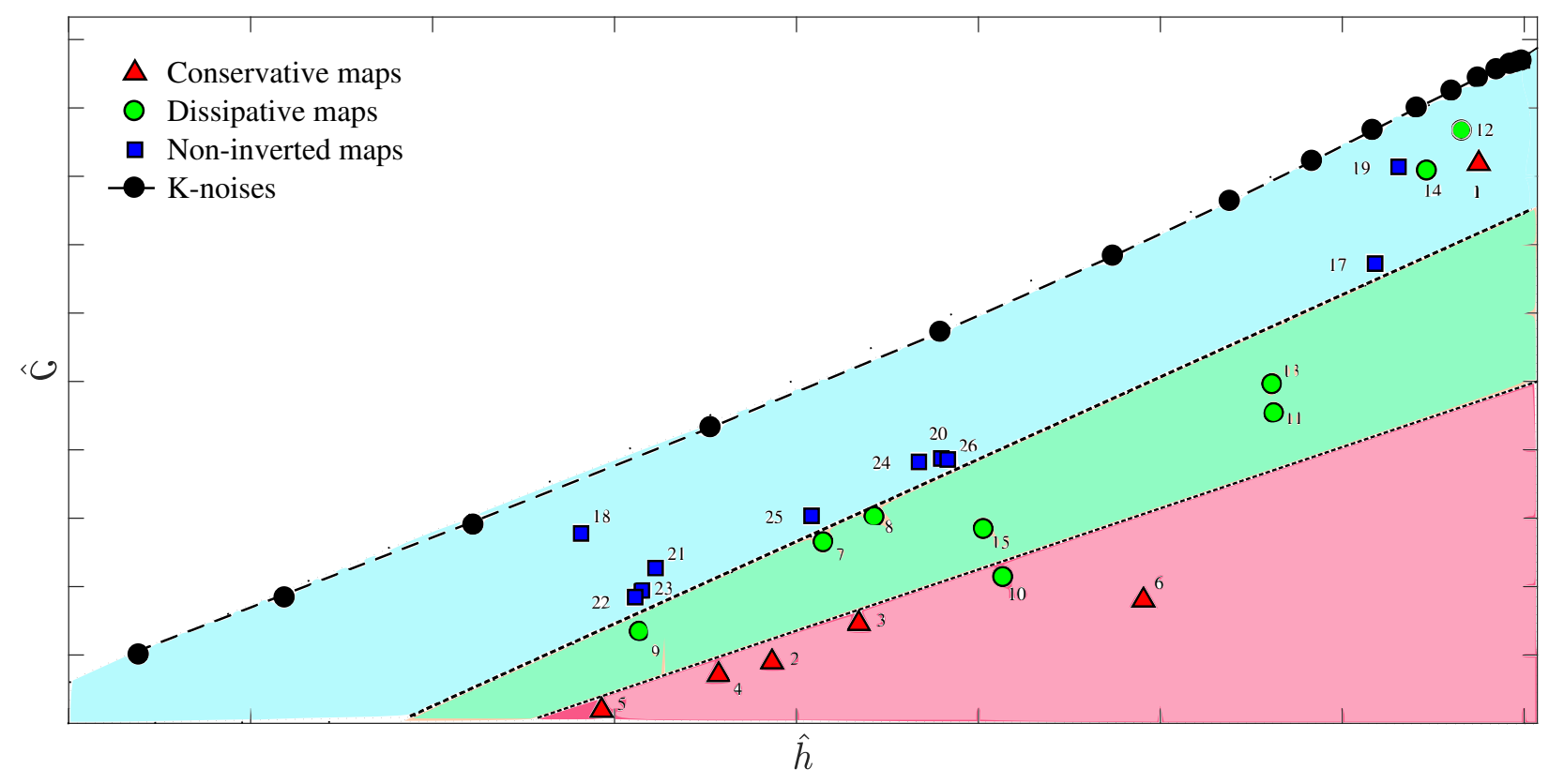

Figure 4: Using the $\widehat{c} \times \widehat{h}$ plane, it is possible to separate the chaotic maps into three zones according to their nature. The blue band lies the non-invertible maps. Below, in the green area, resides most of the dissipative maps except Ikeda map (14) and the dissipative standard map (12). In inferior triangle stay distributed the conservative maps which have the lower complexity values.

to the incorporation of an algorithmic measure such as the Lempel-Ziv complexity, and is based on that for LTM need a greater amount of code to represent the signal than STM, this characteristic is not detectable with statistical measures used by others authors. It is important to mention a plane introduced by Tarnopolsky [83] based on variance and turning points of a time series can also distinguish between FBM from FGN but chaotic dynamics as Lorenz map or Chirkov map are difficult to differentiate from FBM, which is not our case. In our EC-plane these two dynamics are well separated. For the non-Gaussian noises $(K$-noise) can be observed as the correlation increases entropy and complexity decrease, tending to reside on the limit line.

The distribution of the complexity and entropy values in the plane allows to differentiate between deterministic and stochastic processes, since the deterministic process is always below the straight line where the $K$-noises reside. Therefore, our permutation Lempel-Ziv complexity vs permutation Shannon entropy plane is a "robust tool to distinguish chaotic from random signals, as well as to observe the degree of correlation existing in the signal. Due to all the properties mention above, this EC-plan could be applied in many fields from biology, medicine to engineering.

As future direction of investigation, the maps proposed in the literature should be compared through automatic classification approaches. One can also imagine combining three measures to capture more complementary aspects rather than only "permutation" statistical and algorithmic aspects, without a too complex estimation/evaluation procedure. Similarly, rather than Shannon entropy, generalized entropy may be able to capture finer statistical 
aspects ( e.g., the tails of head of distribution via Rényi-Tsallis entropies).

As a future direction of investigation, the maps proposed in the literature should be compared through automatic classification approaches. One can also imagine combining three measures to capture more complementary aspects rather than only "permutation statistical and algorithmic aspects, without a too complex estimation/evaluation procedure. Similarly, rather than Shannon entropy, generalized entropy may capture finer statistical aspects ( e.g., the tails of head of distribution via Renyi-Tsallis entropies).

\section{References}

[1] W. Herman, A study in the analysis of stationary time series, Ph.D. thesis, Almqvist \& Wiksell (1938).

[2] S. Cambanis, C. D. Hardin, A. Weron, Innovations and Wold decompositions of stable sequences, Probability theory and related fields 79 (1) (1988) 1-27.

[3] T. M. Cover, J. A. Thomas, Elements of Information Theory, 2nd Edition, John Wiley \& Sons, Hoboken, New Jersey, 2006.

[4] R. Q. Quiroga, J. Arnhold, K. Lehnertz, P. Grassberger, Kulback-Leibler and renormalized entropies: Applications to electroencephalograms of epilepsy patients, Physical Review E 62 (6) (2000) 8380-8386. doi:10.1103/PhysRevE.62.8380.

[5] O. A. Rosso, S. Blanco, J. Yordanova, V. Kolev, A. Figliola, M. Schürmann, E. Başar, Wavelet entropy: a new tool for analysis of short duration brain electrical signals, Journal of neuroscience methods 105 (1) (2001) 65-75. doi:10.1016/S0165-0270(00)00356-3.

[6] T. Schreiber, Measuring information transfer, Physical Review Letters 85 (2) (2000) 461-464. doi:10.1103/PhysRevLett.85.461.

[7] M. Rajković, Extracting meaningful information from financial data, Physica A 287 (3-4) (2000) 383395. doi:10.1016/S0378-4371(00)00377-0.

[8] A. Wolf, J. B. Swift, H. L. Swinney, J. A. Vastano, Determining Lyapunov exponents from a time series, Physica D 16 (3) (1985) 285-317. doi:10.1016/0167-2789(85)90011-9.

[9] R. Nagarajan, Quantifying physiological data with lempel-ziv complexity-certain issues, Biomedical Engineering, IEEE Transactions on 49 (11) (2002) 1371-1373.

[10] M. Aboy, R. Hornero, D. Abásolo, D. Álvarez, Interpretation of the Lempel-Ziv complexity measure in the context of biomedical signal analysis, IEEE Transactions on Biomedical Engineering 53 (11) (2006) 2282-2288. doi:10.1109/TBME.2006.883696.

[11] S. Zozor, P. Ravier, O. Buttelli, On Lempel-Ziv complexity for multidimensional data analysis, Physica A 345 (1-2) (2005) 285-302. doi:10.1016/j.physa.2004.07.025.

[12] C. Vignat, J. Bercher, Analysis of signals in the Fisher-Shannon information plane, Physics Letters A 312 (1-2) (2003) 27-33. doi:10.1016/S0375-9601(03)00570-X.

[13] O. A. Rosso, H. A. Larrondo, M. T. Martin, A. Plastino, M. A. Fuentes, Distinguishing noise from chaos, Physical Review Letters 99 (15) (2007) 154102. doi:10.1103/PhysRevLett.99.154102.

[14] O. A. Rosso, F. Olivares, L. Zunino, L. D. Micco, A. L. L. Aquino, A. Plastino, H. A. Larrondo, Characterization of chaotic maps using the permutation Bandt-Pompe probability-distribution, The European Physics Journal B 86 (4) (2013) 116-128. doi:10.1140/epjb/e2013-30764-5.

[15] O. A. Rosso, H. Craig, P. Moscato, Shakespeare and other English renaissance authors as characterized by information theory complexity quantifiers, Physica A 388 (6) (2009) 916-926. doi:10.1016/j.physa.2008.11.018.

[16] A. F. Bariviera, L. Zunino, O. A. Rosso, An analysis of high-frequency cryptocurrencies prices dynamics using permutation-information-theory quantifiers, Chaos: An Interdisciplinary Journal of Nonlinear Science 28 (7) (2018) 075511.

[17] F. Montani, O. A. Rosso, Entropy-complexity characterization of brain development in chickens, Entropy 16 (8) (2014) 4677-4692. doi:10.3390/e16084677. 
[18] P. W. Lamberti, M. T. Martin, A. Plastino, O. A. Rosso, Intensive entropic non-triviality measure, Physica A 334 (1-2) (2004) 119-131. doi:10.1016/j.physa.2003.11.005.

[19] M. T. Martin, A. Plastino, O. A. Rosso, Generalized statistical complexity measures: Geometrical and analytical properties, Physica A: Statistical Mechanics and its Applications 369 (2) (2006) 439-462.

[20] A. Lempel, J. Ziv, On the complexity of finite sequences, IEEE Transactions on Information Theory 22 (1) (1976) 75-81. doi:10.1109/TIT.1976.1055501.

[21] C. E. Shannon, A mathematical theory of communication, The Bell System Technical Journal 27 (4) (1948) 623-656. doi:10.1002/j.1538-7305.1948.tb00917.x.

[22] L. Zunino, M. C. Soriano, I. Fischer, O. A. Rosso, C. R. Mirasso, Permutation-information-theory approach to unveil delay dynamics from time-series analysis, Physical Review E 82 (4) (2010) 046212. doi:10.1103/PhysRevE.82.046212.

[23] J. Beirlant, E. J. Dudewicz, L. Györfi, E. C. van der Meulen, Nonparametric entropy estimation: An overview, International Journal of Mathematical and Statistical Sciences 6 (1) (1997) 17-39.

[24] N. Leonenko, L. Pronzato, V. Savani, A class of Rényi information estimators for multidimensional densities, Annals of Statistics 36 (5) (2008) 2153-2182. doi:10.1214/07-AOS539.

[25] T. Schürmann, P. Grassberger, Entropy estimation of symbol sequences, Chaos 6 (3) (1996) 414. doi:10.1063/1.166191.

[26] A. O. Hero III, B. Ma, O. J. J. Michel, J. Gorman, Application of entropic spanning graphs, IEEE Signal Processing Magazine 19 (5) (2002) 85-95. doi:10.1109/MSP.2002.1028355.

[27] S. Frenzel, B. Pompe, Partial mutual information for coupling analysis of multivariate time series, Physical Review Letters 99 (20) (2007) 204101. doi:10.1103/PhysRevLett.99.204101.

[28] M. Rosenblatt, Remarks on some nonparametric estimates of a density function, The Annals of Mathematical Statistics 27 (3) (1956) 832-837.

[29] E. Parzen, On estimation of a probability density function and mode, The Annals of Mathematical Statistics 33 (3) (1962) 1065-1076.

URL http://www.jstor.org/stable/2237880

[30] C. Bandt, B. Pompe, Permutation entropy: A natural complexity measure for time series, Physical Review Letters 88 (17) (2002) 174102. doi:10.1103/PhysRevLett.88.174102.

[31] S. Zozor, D. Mateos, P. W. Lamberti, Mixing Bandt-Pompe and Lempel-Ziv approaches: another way to analyze the complexity of continuous-states sequences, The European Physical Journal B 87 (5) (2014) 107. doi:10.1140/epjb/e2014-41018-5.

[32] J. B. Gao, J. Hu, W. W.Tung, Y. H. Cao, Distinguishing chaos from noise by scale-dependent lyapunov exponent, Physical Review E 74 (6) (2006) 066204.

[33] L. B. (translated by Stephen G. Brush), Lectures on Gas Theory, Dover, Leipzig, Germany, 1964.

[34] J. W. Gibbs, Elementary Principle in Statistical Mechanics, University Press - John Wilson and son, Cambridge, USA, 1902.

[35] J. von Neumann, Thermodynamik quantenmechanischer gesamtheiten, Nachrichten von der Gesellschaft der Wissenschaften zu Göttingen 1 (1927) 273-291.

[36] F. R. S. W. D. Nieven, M. A., The scientific papers of James Clerk Maxwell, Vol. 2, Dover, New-York, 1952.

[37] E. T. Jaynes, Gibbs vs Boltzmann entropies, American Journal of Physics 33 (5) (1965) 391-398. doi:10.1119/1.1971557.

[38] I. Müller, W. H. Müller, Fundamentals of Thermodynamics and Applications. With Historical Annotations and Many Citations from Avogadro to Zermelo, Springer, Berlin, 2009. doi:10.1007/978-3-54074648-5.

[39] M. Planck, Eight Lectures on Theoretical Physics, Columbia University Press, New-York, 2015.

[40] A. I. Khinchin, Mathematical foundations of information theory, Dover Publications, New-York, 1957.

[41] J. Ziv, A. Lempel, A universal algorithm for sequential data compression, IEEE Transactions on Information Theory 23 (3) (1977) 337-343. doi:10.1109/TIT.1977.1055714.

[42] J. Ziv, A. Lempel, Compression of individual sequences via variable-rate coding, IEEE Transactions on Information Theory 24 (5) (1978) 530-536. doi:10.1109/TIT.1978.1055934. 
[43] A. D. Wyner, J. Ziv, Some asymptotic properties of the entropy of a stationary ergodic data source with applications to data compression, IEEE Transactions on Information Theory 35 (6) (1989) 1250-1258. doi:10.1109/18.45281.

[44] G. Hansel, Estimation of the entropy by the Lempel-Ziv method, Lecture Notes in Computer Science (Electronic Dictionaries and Automata in Computational Linguistics) 377 (1989) 51-65. doi:10.1007/3540-51465-1-4.

[45] F. Kaspar, H. G. Schuster, Easily calculable measure for the complexity of spatiotemporal patterns, Physical Review A 36 (2) (1987) 842-848. doi:10.1103/PhysRevA.36.842.

[46] F. Takens, Detecting strange attractors in turbulence, in: D. Rand, L.-S. Young (Eds.), Dynamical Systems and Turbulence, Vol. 898 of Lecture Notes in Mathematics, Springer Verlag, Warwick, 1981, pp. 366-383. doi:10.1007/bfb0091924.

[47] J. C. Robinson, Dimensions, Embeddings, and Attractors, Cambridge University Press, Cambdrige, UK, 2011.

[48] J. M. Amigó, L. Kocarev, J. Szczepanski, Order patterns and chaos, Physics Letters A 355 (1) (2006) 27-31. doi:10.1016/j.physleta.2006.01.093.

[49] J. M. Amigó, Permutation Complexity in Dynamical Systems, Springer Verlag, Heidelberg, 2010.

[50] J. M. Amigó, S. Zambrano, M. A. F. Sanjuán, True and false forbidden patterns in deterministic and random dynamics, Europhysics Letters 79 (5) (2007) 50001. doi:10.1209/0295-5075/79/50001.

[51] O. A. Rosso, L. C. Carpi, P. M. Saco, M. Gómez Ravetti, Causality and the entropycomplexity plane: Robustness and missing ordinal patterns, Physica A 391 (1-2) (2012) 42-45. doi:10.1016/j.physa.2011.07.030.

[52] J. Sprott, Chaos and time-series analysis, Oxford University Press, Oxford, 2003.

[53] P. Dutta, P. M. Horn, Low-frequency fluctuations in solids: $1 / f$ noises, Reviews of Modern Physics 53 (3) (1981) 497-516. doi:10.1103/RevModPhys.53.497.

[54] J. R. M. Hosking, Fractional differencing, Biometrika $68 \quad(1) \quad(1981) \quad 165-176$. doi:10.1093/biomet/68.1.165.

[55] B. West, M. Shlesinger, The noise in natural phenomena, American Scientist 78 (1) (1990) 40-45. URL http://www. jstor.org/stable/29773862 .

[56] A. N. Kolmogorov, Sienersche spiralen und einige andere interessante kurven im hilbertschen raum, Doklady Akademii nauk SSSR 26 (2) (1940) 115-118.

[57] H. Hurst, Long-term storage capacity in reservoirs, Transactions of the American Society of Civil Engeniering 116 (1951) 770-799.

[58] B. Mandelbrot, J. W. Van Ness, Fractional Brownian motions, fractional noises and applications, SIAM review 10 (4) (1968) 422-437. doi:10.1137/1010093.

[59] L. Zunino, D. G. Pérez, M. T. Martín, A. Plastino, M. Garavaglia, O. A. Rosso, Characterization of Gaussian self-similar stochastic processes using wavelet-based informational tools, Physical Review E 75 (2) (2007) 021115. doi:10.1103/PhysRevE.75.021115.

[60] P. Flandrin, On the spectrum of fractional Brownian motions, IEEE Transactions on Information Theory 35 (1) (1989) 197-199. doi:10.1109/18.42195.

[61] J. Beran, Statistics for Long-Memory Processes, Chapman \& Hall, New-York, 1994.

[62] G. Samorodnitsky, M. S. Taqqu, Stable Non-Gaussian Random Processes. Stochastic Models with infinite Variance, Chapman \& Hall, New-York, 1994.

[63] J. Feder, Fractals, Vol. 9, Springer, New-York, 1988. doi:10.1007/978-1-4899-2124-6.

[64] F. J. Molz, H. H. Lui, J. Szulga, Fractional Brownian motion and fractional Gaussian noise in subsurface hydrology: A review, presentation of fundamental properties, and extensions, Water Resources Research 33 (10) (1997) 2273-2286. doi:10.1029/97WR01982.

[65] G. Samorodnitsky, Long range dependence, Foundations and Trends ${ }^{\circledR}$ in Stochastic Systems 1 (3) (2007) 163-257. doi:10.1561/0900000004.

[66] R. B. Davies, D. S. Harte, Tests for Hurst effect, Biometrika 74 (1) (1987) 95-101. doi:10.1093/biomet/74.1.95.

[67] P. Abry, F. Sellan, The wavelet-based synthesis for fractional Brownian motion proposed by F. Sellan 
and Y. Meyer: Remarks and fast implementation, Applied and Computational Harmonic Analysis 3 (4) (1996) 377-383. doi:10.1006/acha.1996.0030.

[68] W. Kinzel, G. Reents, Physics by Computers - Programming Physical Problems Using Mathematica ${ }^{\circledR}$ and C, Springer Verlag, Heidelberg, 1998.

[69] L. Zunino, D. G. Pérez, A. Kowalski, M. T. Martín, M. Garavaglia, A. Plastino, O. A. Rosso, Fractional Brownian motion, fractional Gaussian noise, and Tsallis permutation entropy, Physica A: Statistical Mechanics and its Applications 387 (24) (2008) 6057-6068.

[70] L. Zunino, M. C. Soriano, O. A. Rosso, Distinguishing chaotic and stochastic dynamics from time series by using a multiscale symbolic approach, Physical Review E 86 (4) (2012) 046210.

[71] U. Huebner, N. Abraham, C. Weiss, Dimensions and entropies of chaotic intensity pulsations in a single-mode far-infrared nh 3 laser, Physical Review A 40 (11) (1989) 6354.

[72] W. Wang, J. Vrijling, P. H. Van Gelder, J. Ma, Testing for nonlinearity of streamflow processes at different timescales, Journal of Hydrology 322 (1-4) (2006) 247-268.

[73] B. Sivakumar, Chaos theory in geophysics: past, present and future, Chaos, Solitons \& Fractals 19 (2) (2004) 441-462.

[74] W.-w. Tung, J. Gao, J. Hu, L. Yang, Detecting chaos in heavy-noise environments, Physical Review E 83 (4) (2011) 046210.

[75] United states geological survey, http://www.usgs.gov, accessed: 2010-09-30.

[76] J. M. Hausdorff, P. L. Purdon, C.-K. Peng, Z. Ladin, J. Y. Wei, A. L. Goldberger, Fractal dynamics of human gait: stability of long-range correlations in stride interval fluctuations, Journal of applied physiology 80 (5) (1996) 1448-1457.

[77] F. Olivares, L. Zunino, O. A. Rosso, Quantifying long-range correlations with a multiscale ordinal pattern approach, Physica A: Statistical Mechanics and its Applications 445 (2016) 283-294.

[78] Long-term recordings of gait dynamics, http://physionet.org/physiobank/database/umwdb/.

[79] B. G. Malkiel, The efficient market hypothesis and its critics, Journal of economic perspectives 17 (1) (2003) 59-82.

[80] M. Beben, A. Orłowski, Correlations in financial time series: established versus emerging markets, The European Physical Journal B-Condensed Matter and Complex Systems 20 (4) (2001) 527-530.

[81] T. Di Matteo, T. Aste, M. M. Dacorogna, Scaling behaviors in differently developed markets, Physica A: Statistical Mechanics and its Applications 324 (1-2) (2003) 183-188.

[82] Datastream platform, http://financial.thomsonreuters.com/en/products/tools-applications/tradinginvestment-tools/datastream-macroeconomic-analysis.html.

[83] M. Tarnopolski, On the relationship between the hurst exponent, the ratio of the mean square successive difference to the variance, and the number of turning points, Physica A: Statistical Mechanics and its Applications 461 (2016) 662-673. 